\title{
Haptic Positioning Environment for the Hip Resurfacing Surgery
}

\author{
http://dx.doi.org/10.3991/ijoe.v8iS1.1890 \\ P. Pires, P. Teodoro, J. Martins and J. Sá da Costa \\ TULisbon, Lisbon, Portugal
}

\begin{abstract}
The development of new robotic surgical instruments has brought significant improvements to the execution and outcome of surgical procedures. They allow shorter intraoperative interventions with higher precision of the surgical gesture, which has clear benefits for the patients, surgeons and hospitals. Perhaps the main retardant of the widespread of these new tools is the ability of getting the surgeon's hands-on with the new systems, for both initial evaluation and later on to overcome the initial learning curve. To accelerate this stage, and keep costs to a minimum, simulation tools have been introduced which allow physical surgeon-patient interaction through haptic devices. In this work, we focus on the Hip Resurfacing Surgical Procedure, and develop an interactive virtual environment for the insertion of the hip resurfacing guide wire with high precision and accuracy. The surgical tool used is a robotic arm that allows geometric accuracy and repeatability while the surgeon keeps control over the force exerted on the patient.
\end{abstract}

Index Terms-Biomechatronics, Human robot interaction, Medical robotics, Simulation.

\section{INTRODUCTION}

In the last two decades, haptic systems have been an active area of research, where surgical training environments are an important area of focus for haptic devices. The possibility to train surgeons without the need of cadavers and animals reduces costs and eliminates ethical issues that may arise [1].

In this paper, a haptic system and virtual environment are integrated for simulating the guide wire insertion into the femural head in the hip resurfacing surgical procedure. The haptic device used is the Novint Falcon, and the virtual environment is created in Matlab/Simulink for fast implementation of control algorithms, despite of all the necessary interface development [2]. A modified Hertz Law is used to model the bone-tool interaction force, and a variable impedance force law is used for the virtual surgical robot to guide the surgeon's hand to the correct position over the femur.

This paper is organized as follows. Section II introduces the surgical procedure. Section III presents the hardware interface along with the environment force fields. Results are presented in Section IV, followed by conclusions in Section V.

\section{PROBLEM STATEMENT}

Total hip resurfacing is a technically demanding surgical procedure and as any other procedures has a significant learning curve. In [3], a study of a single surgeon's

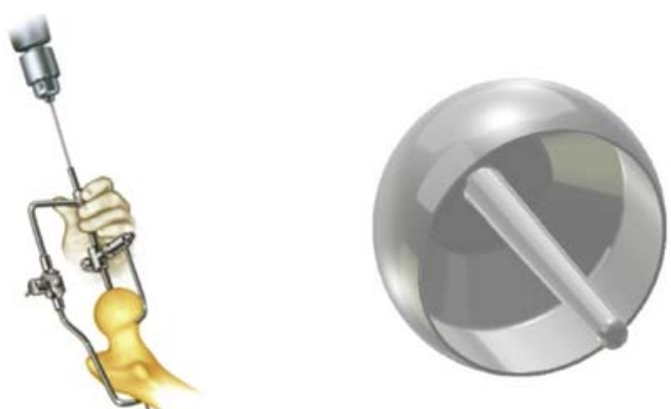

Figure 1. Left - McMinn Alignment Guide, from [4]. Right - Birmingham Hip Resurfacing prosthesis.

learning curve was performed where the quality of the implant position was measured. A relevant conclusion is that there is significant variability on the implant positioning. The position of the femoral implant is of extreme importance for the surgery success. Measurements obtained from the preoperative planning are evaluated in order to obtain the desired position of the femoral guide wire, Fig. 1.

A robotic arm may aid the surgeon to perform the procedure by actively guiding the drill to the correct orientation and position, [5]. It minimizes the risk of incorrect positioning during drilling and a reduction in surgery time is expected, as well as a significant increase in geometrical accuracy and precision, [6].

\section{PRELIMINARIES}

\section{A. Haptic hardware interface}

The haptic system used is the Novint Falcon device that communicates with the host computer through a USB 2.0 port, figure 2. It has three degrees of freedom with force feedback that are associated to the robot end-effector. The user thus has feedback of the exerted reaction force over the simulated patient femoral bone and the applied forces resulting from the control laws implemented. The visualization processing is evaluated in a separate computer from the host computer due to sample time constraints.

\section{B. Force Field Formulation}

The contact model adopted in this work is the recently modified non-linear Hertz law presented in [7] that accommodates smaller coefficients of restitution, $e$.

$$
F_{C_{n}}=k_{n} d_{n}^{\delta}\left(1+\frac{8(1-e)}{5 e} \frac{v_{n}}{v_{n}^{(-)}}\right)
$$




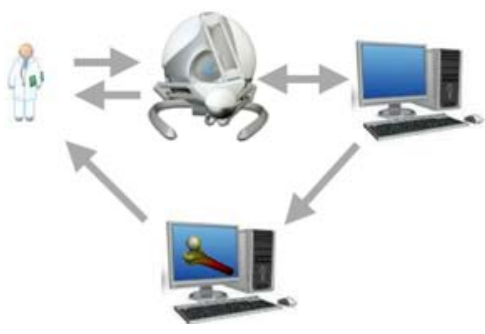

Figure 2. User, Novint Falcon Haptic and PC display interface communications.

$k_{n}$ represents the generalized stiffness constant along the normal direction, $v_{n}^{(-)}$is the velocity at the beginning of the contact, $d_{n}$ the penetration distance along normal direction. In Fig. 3, a virtual cone attached to the drilling point of the femoral head and oriented along the normal drilling direction is presented. This virtual surface is a frictionless guiding surface and the contact force is obtained by (1). $F_{i}$ is the impedance force applied in the drill in order to push it to the $Z_{s}$ axis. The cone surface parameterization is defined as:

$$
\begin{aligned}
& x_{s}=r \cos (\theta), \quad y_{s}=r \sin (\theta) \\
& z_{s}=c \sqrt[4]{\left(\frac{x_{s}}{a}\right)^{2}+\left(\frac{y_{s}}{a}\right)^{2}-1-b}
\end{aligned}
$$

where the parameters $a, b$ and $c$ are constants related with the minimum radius at $Z_{s}=0$, the surface offset and the cone opening scale. When there is no contact with the surface the drill stays in gravity compensation. To guide the virtual end-effector position towards the bone axis $Z_{s}$ an impedance environment is superimposed. The first order system that relates the radial displacement $r$, with the impedance force $F_{i}$ has the following Laplace Transform:

$$
\frac{R(s)}{F_{i}(s)}=\frac{1}{b s+K}=\frac{1 / K}{\tau s+1}
$$

where the time constant is $\tau=0.05 s$ and the stiffness $K$ increases with the decrease of the axial displacement $Z_{s}$.

\section{RESULTS}

For demonstration, some experiments were made to test the control methodology. In Fig. 4 the impedance force on the left and the surface reaction force on the right are related with the radial displacement error at the center. With small effort, the tool is guided to the correct position as we approach to the bone drilling point.

\section{CONCLUSION}

A haptic environment was developed for training surgeons in robotic assisted total hip resurfacing surgery using low cost hardware. The implemented control algorithm guides the surgeon to the correct position by force feedback in a physical surgeon-robot interaction environment.

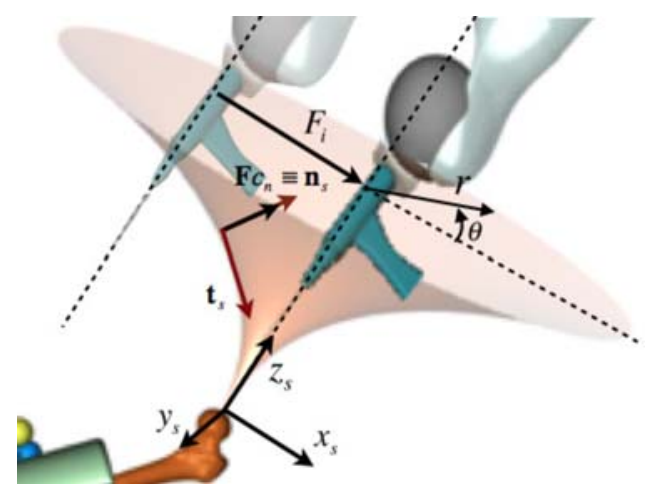

Figure 3. Haptic virtual environment.

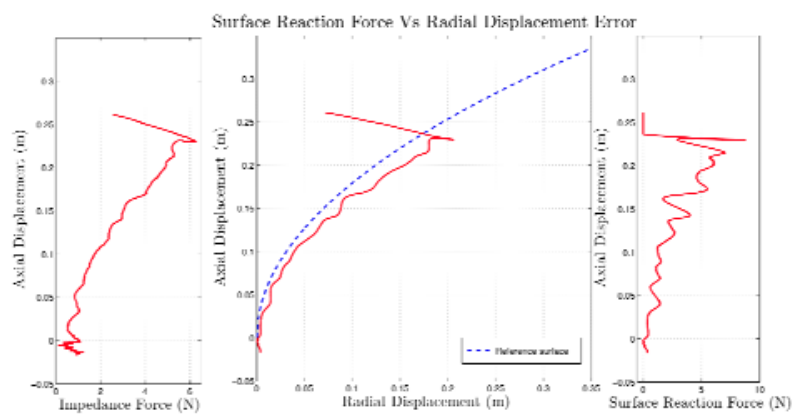

Figure 4. Surface Reaction Force and Impedance Force versus Radial Displacement Error.

\section{ACKNOWLEDGMENT}

This work was supported by FCT-Fundação para a Ciência e a Tecnologia, through project PTDC/EME$\mathrm{CRO} / 114571 / 2009$. IDMEC is funded by FCT in the framework of the associated laboratory LAETA. The 1st and 2nd author are supported by FCT PhD sponsorships SFRH/BD/36866/2007 and SFRH/BD/44801/2008 respectively.

\section{REFERENCES}

[1] F. G. Hamza-Lup, C. M. Bogdan, D. M. Popovici, and O. D. Costea, "A survey of visuo-haptic simulation in surgical training," in International Conference on Mobile, Hybrid, and On-line Learning, February 2011.

[2] K. Salisbury, F. Conti, and F. Barbagli, "Haptic rendering: Introductory concepts," Ieee Computer Graphics and Applications, vol. 24, no. 2, pp. 24-32, 2004. http://dx.doi.org/10.1109/MCG.2004. 1274058

[3] S. Witjes, J. Smolders, P. Beaule', P. Pasker, and J. Van Susante, "Learning from the learning curve in total hip resurfacing: a radiographic analysis," Archives of Orthopaedic and Trauma Surgery, vol. 129 , pp. 1293-1299, 2009, http://dx.doi.org/10.1007/s00402009-0875-z

[4] Smith\&Nephew, Birmingham Hip Resurfacing System, Smith\&Nephew, January 2007.

[5] P. Pires, P. Teodoro, J. M. Martins, and J. S. da Costa, "Position and force control of a flexible robot manipulator for orthopedic surgery," in European Control Conference 2009 - ECC'09, Budapest, Hungary, 2009.

[6] R. Bareeq, S. Jayaraman, B. Kiaii, C. Schlachta, J. Denstedt, and S. Pautler, "The role of surgical simulation and the learning curve in robot-assisted surgery," Journal of Robotic Surgery, vol. 2, pp. 11-15, 2008, http://dx.doi.org/10.1007/s11701-008-0074-x

[7] P.Flores,M.Machado,M.T.Silva,andJ.M.Martins, "Onthecontinuous contact force models for soft materials in multibody dynamics," Multibody System Dynamics, vol. 25, no. 3, pp. 357-375, dec 2010. http://dx.doi.org/10.1007/s11044-010-9237-4 


\section{AUTHORS}

P. Pires is with the IDMEC/IST, TULisbon, Lisbon, Portugal (e-mail: pedropires@ ist.utl.pt).

P. Teodoro is with the IDMEC/IST, TULisbon, Lisbon, Portugal (e-mail: pedroteodoro@ist.utl.pt).

J. Martins, is with the IDMEC/IST, TULisbon, Lisbon, Portugal (e-mail: jorgemartins@ist.utl.pt).
J. Sá da Costa is with the IDMEC/IST, TULisbon, Lisbon, Portugal (e-mail: sadacosta@dem.ist.utl.pt).

This work is a description of a demonstration given during $1^{\text {st }}$ Experiment@ International Conference, 17/18 November 2011 in Lisbon, Portugal. It was supported by Calouste Gulbenkian Foundation, Lisbon, Portugal. Manuscript received 01 December 2011. Published as resubmitted by the authors 20 January 2012. 